\title{
Accurate 3D Fingerprint Virtual Environment for Biometric Technology Evaluations and Experiment Design
}

\author{
Ruggero Donida Labati, Angelo Genovese, Vincenzo Piuri, Fabio Scotti \\ Department of Computer Science, Università degli Studi di Milano \\ Milano, 20122, Italy. \\ \{ruggero.donida, angelo.genovese, vincenzo.piuri, fabio.scotti\}@unimi.it
}

\begin{abstract}
Three-dimensional models of fingerprints obtained from contactless acquisitions have the advantages of reducing the distortion present in traditional contact-based samples and the effects of dirt on the finger and the sensor surface. Moreover, they permit to use a greater area for the biometric recognition.

The design and test of three-dimensional reconstruction algorithms and contactless recognition methods require the collection of large databases. Since this task can be expensive and timeconsuming, some methods in the literature deal with the generation of synthetic biometric samples. At the best of our knowledge, however, there is only a preliminary study on the computation of small areas of synthetic three-dimensional fingerprints.

In this paper, we extend our previous work and describe a virtual environment for the generation of complete threedimensional fingertip shapes, which can be useful for the research community working in the field of three-dimensional fingerprint biometrics. The method is based on image processing techniques and algorithms designed for biometric recognition. We validated the realism of the simulated models by comparing them with real contactless acquisitions. Results show that the method is feasible and produces realistic three-dimensional samples which can effectively be processed by biometric recognition algorithms.
\end{abstract}

Index Terms-virtual environment, fingerprint, threedimensional, contactless, experimental design.

\section{INTRODUCTION}

In the context of fingerprint recognition systems, the use of contactless three-dimensional fingerprint reconstruction methods can overcome limitations of traditional contact-based sensors. In particular, it is possible to reduce distortions, effects of the dirt present on the surface of the finger, and there is the possibility to obtain metric and accurate fingerprints in a contactless manner. Moreover, three-dimensional models can increase the area usable for biometric recognition with respect to contact-based acquisitions. However, contactless fingerprint acquisitions present a lower contrast between ridges and valleys, reflections, and a more complex background. The skin itself, in fact, is part of the background, and light conditions strongly influence the acquisitions.

The design and performance evaluation of biometric systems require the collection of big and heterogeneous sets of samples. This process is time consuming and can be expensive. The use of datasets of synthetic traits can therefore reduce the time and cost needed to realize biometric systems.

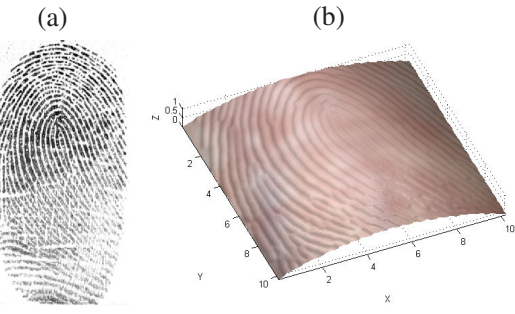

Fig. 1. Synthetic three-dimensional fingerprint presented in [1]. The simulated area is a central $5 \times 5 \mathrm{~mm}$ portion of the fingertip: (a) contactbased acquisition; (b) corresponding three-dimensional synthetic fingerprint.

Also the design and testing of the acquisition setup is a very time-consuming and expensive task since it requires a large numbers of optical and illuminotecnic systems to be tested in different configurations in order to find the optimal configuration with respect to the envisioned application. The adoption of a virtual environment capable to simulate the different configurations of the experimental setup can be of great help to reduce the number of configurations of the real setup to be investigated.

In the literature, several virtual environments have been proposed with the purpose of testing computer vision algorithms [1-3].

While there are several methods in the literature for the computation of real three-dimensional models of the fingerprint [4-8], there is no publicly available database of threedimensional samples. In this context, our method could be useful in providing a ground truth for the evaluation of threedimensional fingerprint recognition methods.

In the context of fingerprint recognition systems, there are different studies on techniques for the simulation of contactbased acquisitions [9-15]. However, there is only a preliminary study on the computation of small portions of contactless three-dimensional samples [1], which presents a method for the simulation of the three-dimensional shape of the ridges and the skin color of an area of $5 \times 5 \mathrm{~mm}$ centered in the core point (Fig. 1). The method also assumes that the finger has a cylindrical shape.

In this paper, we extend the work described in [1] by proposing a virtual environment for the simulation of the complete three-dimensional shape of the fingertip. Starting from real or synthetic images of contact-based acquisitions, the 
presented method permits to obtain realistic synthetic threedimensional models of the finger shape.

The presented method can be divided into five steps. First, the average silhouette is computed from real contactless acquisitions and adapted to the contact-based image, then the three-dimensional shape of the finger is computed by using a parametric model. In the third step, the three-dimensional height of the ridge pattern is estimated and superimposed on the three-dimensional finger shape by using techniques based on empirical analyses of the human morphology. In the fourth step, the camera focus, skin color and illumination conditions are simulated. As a last step, the calibration data of a real system is used to simulate a multiple view acquisition.

\section{PREVIOUS WORKS}

In the literature, many approaches have been described for the computation of synthetic fingerprint images that resemble contact-based acquisitions [9-15].

The technique described in [9] considers global and local features of the fingerprint separately. Global features are related the orientation of the ridge pattern, which is computed using a two-dimensional spatial function described by a limited number of coefficients. The function is then approximated using a second-order model. The local features are initialized as a set of points, and then refined by convoluting them with a stripe-shaped filter, oriented according to the ridge orientation.

A biologically inspired method is proposed in [10,11]. This method is based on the principle that the ridges of real fingers have been formed according to the differential growth of the basal layer of the skin, caused by the compression of the other layers of the skin. This compression is then modeled and used to generate synthetic fingerprints.

A genetic algorithm is used in [12] to generate synthetic fingerprints starting from a set of real fingerprint acquisitions. The genetic algorithm initializes a set of filters, which are used to modify the fingerprint images and generate synthetic samples.

The technique proposed in [13] computes different images describing the area, orientation, and frequency of the ridges. A silhouette is used to describe the area, and the orientation image is obtained by defining a set of singular points and using a flow model to fill the remaining information of the ridge information. The frequency of the pattern is computed by using a criteria defined from observation of real acquisitions. Then, a procedure based on Gabor filtering is applied to generate the actual pattern and the minutiae. Finally, non ideal conditions such as distortion and skin condition are simulated.

The method proposed in [15] is based on the use of real fingerprint acquisitions for the computation of statistical models of features such as singular points, orientation field, and minutiae. The models are computed for each of the main fingerprint classes, such as arch, tented arch, left-loop, right loop, and whorl [16]. A synthetic fingerprint is then generated by choosing a fingerprint type and area, sampling the respective features from the corresponding statistical models, and using a fingerprint reconstruction algorithm.
The only method that proposes the generation of synthetic three-dimensional models of fingerprints is described in [1]. The method uses contact-based acquisition in order to extract the information related to the ridge pattern, and computes the three-dimensional height of the ridges according to observation of real fingerprints. Then, a parametric model is used to generate the shape of the central area of the fingertip and the ridge pattern is superimposed on it. Finally, illumination, color, and blur effects are introduced.

\section{THE DESCRIBED APPROACH}

The proposed approach describes an enhancement over the previous work for the generation of synthetic threedimensional fingerprint models [1]. In particular, the novelties of this method consist in the modeling of the complete shape of the finger, in an enhanced out-of-focus effect, in a more realistic simulation of the illumination, and in the simulation of multiple-view acquisition systems. The approach is based on image processing techniques and algorithms for biometric feature extraction.

In particular, the proposed method uses a contact-based fingerprint image (live, rolled, or synthetic) to obtain the information of the ridge pattern. Then, the silhouette of the fingertip is computed and the three-dimensional finger shape is modeled. The three-dimensional height of the ridges is computed and superimposed on the computed shape. Realistic effects such as noise, pores, incipient ridges, and camera focus are introduced. Then, color information and illumination conditions are introduced. The virtual environment also allows to simulate a contactless biometric acquisition of the simulated three-dimensional models in a multiple view camera system.

The method can be divided into the following steps:

- computation of the finger silhouette;

- computation of the three-dimensional shape of the fingertip;

- computation of the three-dimensional height of the ridges;

- simulation of color and illumination;

- simulation of a multiple view acquisition.

The method is outlined in Fig. 2

\section{A. Computation of the silhouette of the fingertip}

The proposed method computes the three-dimensional finger shape based on the finger silhouette, which is then particularly important for the computation of realistic models. In order to obtain realistic results, the method estimates the silhouette by adapting finger shape models obtained from real contactless acquisitions. The models are computed offline and are different for every finger class. In particular, real fingers have been divided into three categories: thumb, little finger, and other fingers. For each class of fingers, the average silhouette is estimated once offline and it is then used during every computation of synthetic fingerprint models.

In order to compute the average finger silhouettes, real contactless samples are first segmented by using the method described in [7]. 


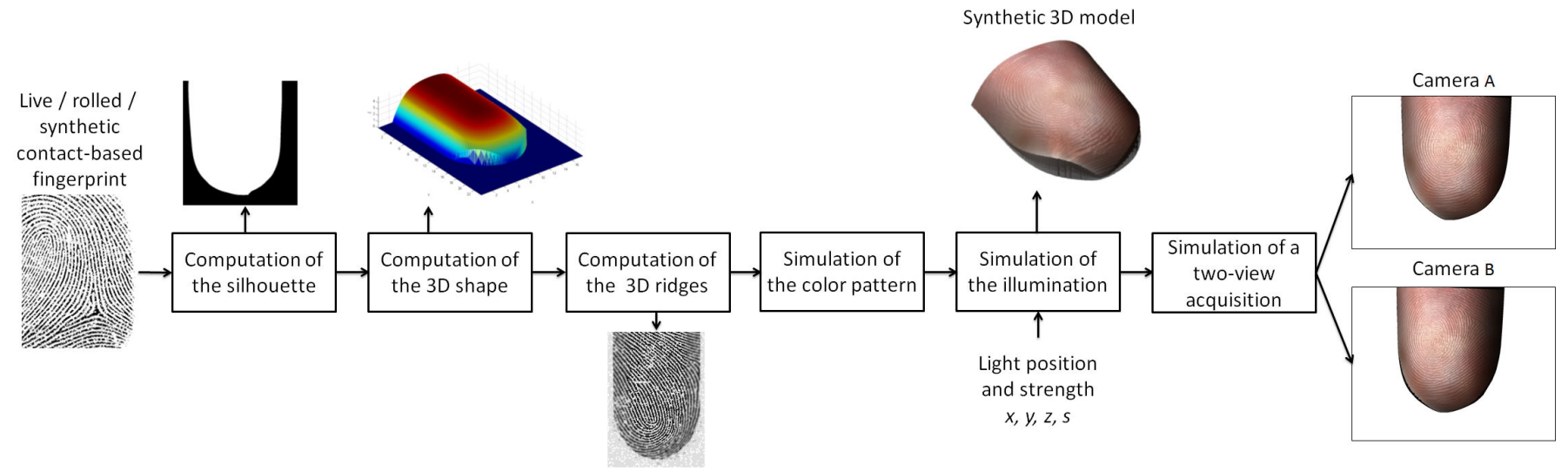

Fig. 2. Outline of the proposed approach for the computation of realistic three-dimensional synthetic fingertips starting from contact-based fingerprint images.

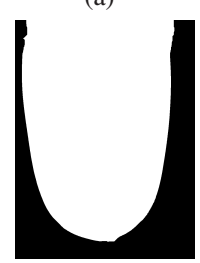

(b)

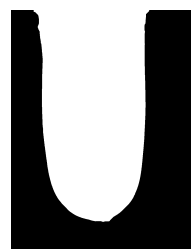

(c)

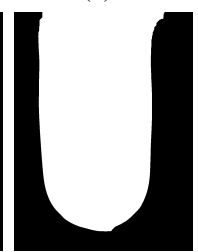

Fig. 3. Average finger silhouettes: (a) average thumb silhouette; (c) average little finger silhouette; (c) average silhouette for the remaining fingers.

Then, the obtained segmented images appertaining to the same finger class are aligned in the two-dimensional space. The proposed method first aligns the top of the fingers along the $y$ axis. The mean finger shape is then obtained by distinctly evaluating the left and right halves of the images appertaining to the considered class. For each row, the average column value is computed:

$$
\begin{aligned}
& m_{L}(y)=\frac{1}{N} \sum_{i=1}^{N} I_{L}\left(x_{i}, y\right) \\
& m_{R}(y)=\frac{1}{N} \sum_{i=1}^{N} I_{R}\left(x_{i}, y\right) \quad \forall y,
\end{aligned}
$$

where $m_{L}(y)$ and $m_{R}(y)$ are the mean column values for the row $y$, respectively for the left and right halves of the silhouette $I_{L}$ and $I_{R}$, and $N$ is the number of considered images. The average silhouette $I_{s}$ is then computed by filling the region delimited by the boundary points $m_{L}(y)$ and $m_{R}(y)$. An example of the computed silhouettes is shown in Fig. 3.

In the computation of every synthetic three-dimensional model, the mean silhouette of the considered finger class is used to obtain a realistic binary image of the finger shape $S$. The mean silhouette $I_{S}$ is resized to the maximum width of the contact-based fingerprint image $I_{C}$. Then, $S$ is cropped starting from the top of the phalanx in order to match the area of the contact-based image $I_{C}$.

\section{B. Computation of the three-dimensional shape of the fingertip}

This step computes the three-dimensional shape of the fingertip based on the silhouette $S$ obtained in the previous

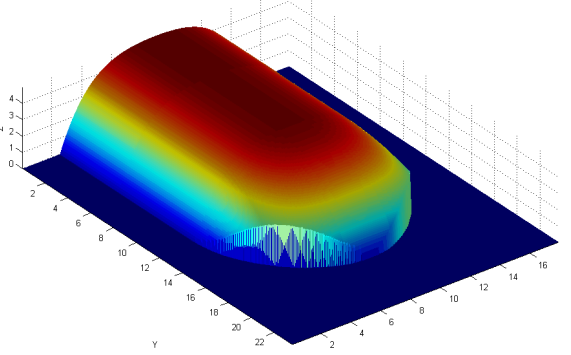

Fig. 4. Example of a synthetic three-dimensional shape of the fingertip.

step. The method is based on the technique described in [17] and computes a parametric finger shape based on fourth-order polynomials. The parametric model has been designed by studying three-dimensional models computed from real acquisitions [7], and permits to create synthetic three-dimensional shape in a simple and accurate manner.

In particular, a fourth order polynomial $p$ is used to approximate the curvature of the finger, by interpolating its values passing by the $x$ and $y$ coordinates:

$$
\begin{array}{r}
\left\{\left(x_{\min }, 0\right),\left(x_{m}-c_{W} x_{m}, c_{H 1}\right),\left(x_{m}, c_{H 2}\right),\right. \\
\left.\left(x_{m}+c_{W} x_{m}, c_{H 1}\right),\left(x_{\max }, 0\right)\right\},
\end{array}
$$

where $x_{\min }$ is the minimum $x$ coordinate of $I_{s}, x_{\max }$ is the maximum $x$ coordinate of $I_{s}, x_{m}=\left(x_{\max }+x_{\min }\right) / 2$, and the values of the parameters $c_{W}, c_{H 1}, c_{H 2}$ are empirically estimated from the real datasets of three-dimensional models.

The finger curvature is then described by the vector $C$, obtained by fitting the polynomial $p$ in the interval $\left(x_{\min }, x_{\max }\right)$, and each column of the depth map $S_{z}$ is obtained as:

$$
S_{z}(i)=I_{s}(i) \times C \times\left(X_{\min }(i)-X_{\max }(i)\right)
$$

where $X_{\min }$ and $X_{\max }$ are the vectors that represent the minimum and maximum values of the $x$ coordinates for each column $i$ of the silhouette $I_{s}$. Fig. 4 shows an example of the computed shape. 


\section{Computation of the three-dimensional height of the ridges}

This step computes the height of the ridges by analyzing the ridge pattern of a real or synthetic contact-based image $I_{C}$ with algorithms commonly used for biometric recognition.

1) Estimation on the height of the ridge pattern: First, the contact-based image $I_{C}$ is aligned with the two-dimensional simulated shape $S$ by using the algorithm described in Section III-A. Then, a first approximation of the three-dimensional ridge shapes $G_{f}$ is performed by using a well-known enhancement method in the literature based on Gabor filters [18], and assuming that the result of the filtering procedure is proportional to the three-dimensional height of the ridges.

The heights of the ridges $R$ and valleys $V$ are refined according to:

$$
R=\log \left(G_{f} \times\left(\neg B_{f}\right)\right) ; V=\log \left(1-G_{f} \times B_{f}\right),
$$

where $B_{f}$ is a binary image obtained from $G_{f}$.

Then, the intensities of the ridges are normalized in the interval $\left(1-\Delta_{r}, 1\right)$, and the intensities of the valleys are normalized in the interval $\left(0, \Delta_{r}\right)$, where $\Delta_{r}$ is tuned to resemble real three-dimensional fingerprints.

The final image $H$, which describes the height of the ridge pattern in a non-dimensional space, is computed as $H=R+V$. An adaptive histogram equalization procedure is then applied to $H$.

Finally, incipient ridges and pores are simulated by summing to $H$ a binary image $B_{n}$, which is obtained by applying morphological operators to a matrix of salt and pepper noise.

2) Simulation of camera focus: The technique proposed in this subsection is different from the one described in [1] and permits to better model the camera focus on a surface with a complex shape.

In order to simulate the out-of-focus on the peripheral regions of the images, the ridge pattern is smoothened progressively towards the borders.

First, five regions $S_{i}, i=1 \ldots 5$ are defined by subtracting from the mask $I_{s}$ the images obtained by eroding the mask using a structural element with increasing size. The size of the structural element is chosen respectively as $1 / 15,1 / 12,1 / 9,1 / 8,1 / 7$ of the width of the mask $I_{s}$.

Each region of the ridge pattern is filtered according to its spatial position using a low-pass Gaussian filter, with proper area and standard deviation:

$$
H_{f}(x, y)=H_{q}(x, y) * G_{i} \text { if }(x, y) \in S_{i} \quad, \forall i=1 \ldots 5,
$$

where $G_{i}$ is a $m_{i} \times m_{i}$ filter with $\sigma_{i}$ standard deviation, with $m_{i}>m_{i+1}$ and $\sigma_{i}>\sigma_{i+1}$. The area and standard deviation of the filters used in each region have been empirically tuned by observing real contactless samples.

An example of the simulation of the camera focus on the ridge pattern is shown in Fig. 5.

3) Superimposition of the ridges: This step aims to merge the information related to the finger shape and the height of the ridge pattern, obtaining a depth map describing the fingerprint model. (a)

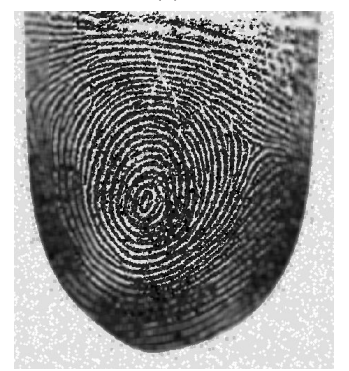

(b)

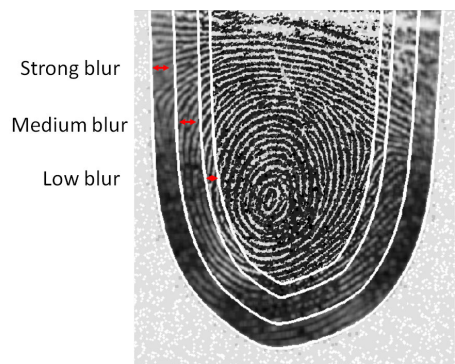

Fig. 5. Graphical example of the simulation of camera focus. For simplicity, only three regions are depicted: (a) ridge pattern; (b) ridge pattern blurred according to the different regions.

First, the coordinates $(x, y)$ of both the ridge pattern $H_{q}$ and the fingertip shape $S_{z}$ are converted to millimeters by considering that the contact-based image $I_{C}$ presents a resolution of 500 dpi. The height values of $H_{q}$ are then adjusted to a mean value of $0.06 \mathrm{~mm}$, corresponding to the mean height of human ridges.

The superimposition of the ridges on the fingertip surface is performed by summing the ridge height $H$ to $S_{z}$ along the direction of its surface normals.

A bilinear interpolation is finally applied in order to obtain a dense representation $S_{r}$ of the fingertip surface.

\section{Simulation of color and illumination}

The color information is computed from a real contactless image $I_{B}$, captured using a technique similar to the one described in [7]. The ridge pattern from $I_{B}$ is removed by using a Gaussian low-pass filter, then the resulting image $I_{p}$ is superimposed on the surface $S_{r}(x, y)$ using a texture mapping procedure.

The proposed illumination model simulates the ambient light, an artificial light source, and realistically simulates the skin reflection. In order to simulate the ambient light, a light source placed at infinity is used to illuminate the scene. Then, a punctiform light source, positioned at coordinates $\left(x_{l}, y_{l}, z_{l}\right)$ and radiating uniformly in all directions, is used to simulate a LED illumination. Specular features of the model are adjusted to match the ones of the human skin, and a Phong lighting algorithm is applied to compute the reflections. Anyway, more complex rendering methods can be used [19].

Some examples of obtained models are shown in Fig. 6.

\section{E. Simulation of a multiple view acquisition}

The proposed virtual environment permits to compute images representing contactless biometric acquisitions of the simulated three-dimensional fingerprint model, performed with a multiple view system.

The implemented method performs three-dimensional projections using calibration data obtained from real acquisition setups [7]:

$$
\left[\begin{array}{lll}
u & v & 1
\end{array}\right]^{T}=K[R \mid t]\left[\begin{array}{llll}
X & Y & Z & 1
\end{array}\right]^{T},
$$

where $(u, v)$ are the projected pixel coordinates, $K$ is the intrinsic camera matrix, $R$ and $t$ are the rotation matrix 
(a)

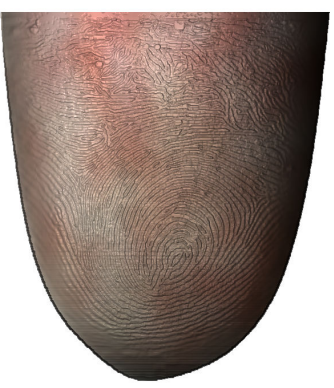

(f)

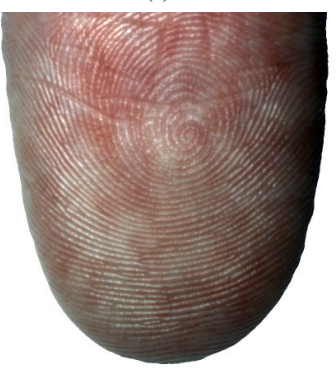

(b)

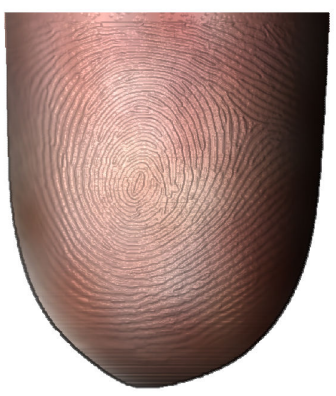

(g)

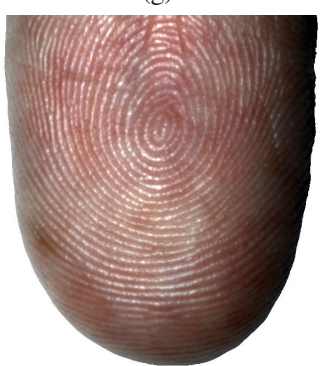

(c)

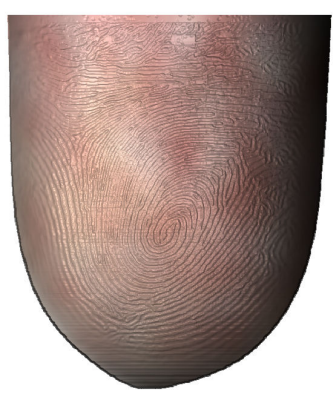

(h)

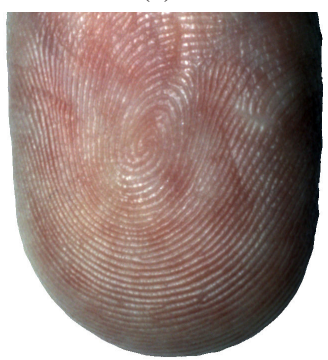

(d)

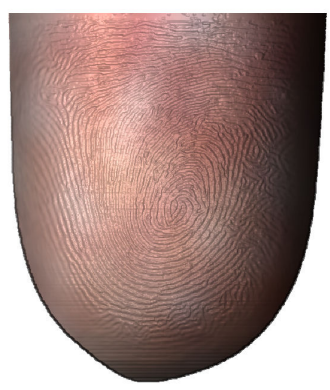

(i)

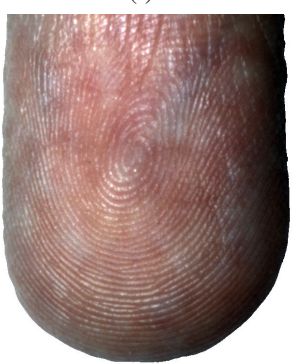

(e)

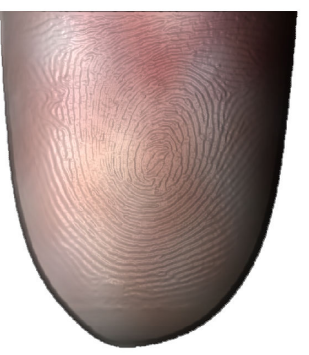

(j)

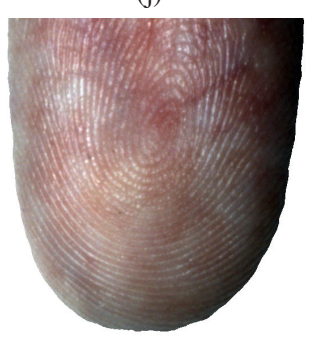

Fig. 6. Examples of the obtained results: (a-e) simulated fingerprint models seen from a top view; (f-j) contactless real images captured from the fingers related to the contact-based images $I_{C}$ used to compute the sinthetic three-dimensional models. The camera is positioned in the simulated point of views used to process the virtual images.

and translation vector. The images related to every view are computed using a linear interpolation.

\section{EXPERIMENTAL RESULTS}

The evaluation of the presented method has been done by applying the simulation algorithms on rolled contact-based fingerprints and comparing the resulting models with the corresponding contactless samples captured using a Sony XCDSX90CR camera [7]. Rolled fingerprints have been chosen over live contact-based fingerprints because they describe also the ridges present on the borders of the fingertip.

The considered real contactless images have a size of $1280 \times$ 960 pixels. The described method has been applied on rolled fingerprints, and the values of the parameters that produce the most realistic results have been searched. In particular, the following values of the parameters for defining the shape of the finger have been used: $c_{W}=3 / 5, c_{H 1}=4, c_{H 2}=5$. The value used to normalize the intensities of the ridges and valleys has been chosen as $\Delta_{r}=0.2$. The sizes and standard deviations of the Gaussian filters, used to smooth the finger borders in the camera focus simulation, have been chosen as: $m=\{10,7,5,3,2\}$ and $\sigma=\{7,5,3,2,1\}$.

The effects of the light sources on the simulated models have been considered in order to achieve visual realism, and the intensities and positions of both the ambient light and local light sources have been adjusted experimentally in order to replicate their effects. Examples of the results are shown in Fig. 6. It is possibile to observe that the computed models are realistic and resemble the real contactless acquisitions.

In order to test the visual realism of the illumination effects, different positions of the punctiform light source have been considered (Fig. 7). The images show that the proposed (a)

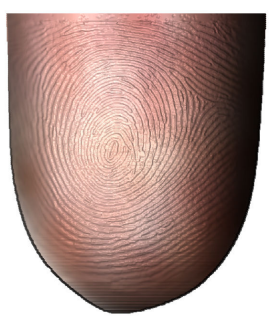

(b)

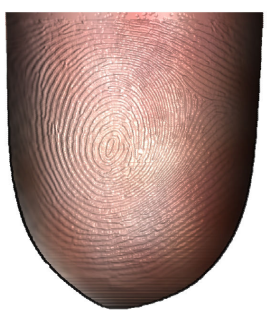

(c)

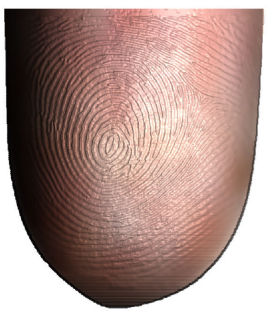

Fig. 7. Examples of synthetic models computed with different position of the punctiform light source: (a) light positioned to the left; (b) light positioned in a central position; (c) light positioned to the right. Results indicate that the presented method can be used to perform quick and effective experiment design activities by simulating different conditions and geometries of the setup (cameras, illumination systems and fingers).

method can effectively simulate different light conditions in a realistic manner.

In order to evaluate the usability of the simulated models for testing biometric recognition algorithms, we have analyzed the results obtained by a well-known minutiae-based algorithm on contact-equivalent images obtained from simulated multiple view acquisitions. The multiple view acquisitions have been simulated using the method described in section III-E, and the three-dimensional reconstruction procedure and unwrapping method described in [7] have been applied. Then, the unwrapped images have been enhanced using the method described in [17] in order to compute the contact-equivalent acquisitions. The obtained images have then been processed by using the minutiae extraction software NIST MINDTCT [20], designed for contact-based images. Examples of the enhanced images and the extracted minutiae are shown in Fig. 8. It is possible to observe that the synthetic samples can be processed using algorithms for the computation of contact-equivalent 
(a)

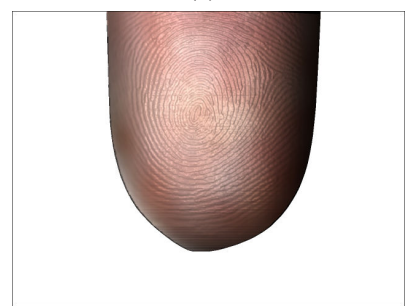

(c)

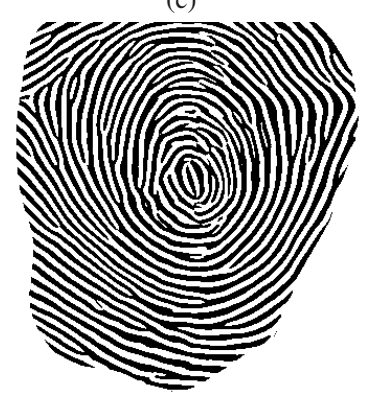

(b)

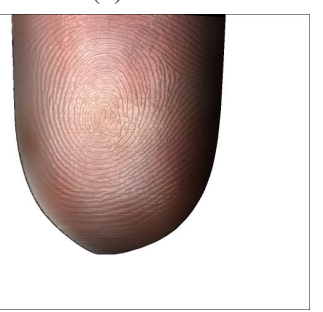

(d)

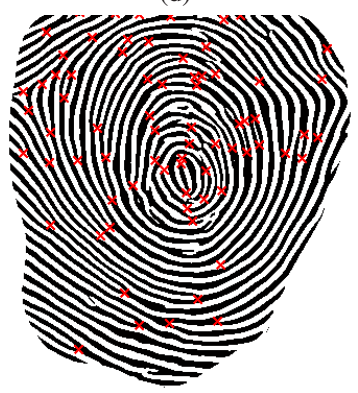

Fig. 8. Simulation of a two-view acquisition, computation of the threedimensional model, unwrapping, and computation of the contact-equivalent images: (a) simulated acquisition obtained by camera A; (b) camera B; (c) contact-equivalent image computed from synthetic model after reconstructing the three-dimensional model from the simulated two-view acquisition and the unwrapping step; (d) extraction of the minutiae from the contact-equivalent image. The results suggest that the synthetic samples obtained by the proposed method can effectivelly be used to test biometric recognition algorithms.

images from contactless acquisitions, obtaining similar results with respect to real samples. Synthetic models computed by using the presented method can then be used in order to extract the minutiae and perform the biometric recognition.

\section{CONClusion}

In this work, we described an improved virtual environment for the generation of synthetic three-dimensional fingerprint samples. This method helps to decrease the time and efforts needed to create tentative setups during the experiment design (saving time and the cost for expensive optical and light equipment used in the search of the optimal system) and facilitate the collection of large databases, necessary to effectively test the biometric recognition algorithms. It can be considered as a specific and flexible tool for researchers working in the field of three-dimensional fingerprint reconstruction and recognition.

The method uses image processing techniques and biometric recognition algorithms to simulate the three-dimensional shape of the fingertip, the details of the ridge pattern, and different camera and illumination conditions.

The obtained results show that the described method achieves a realistic modeling of the fingertip shape and the fingerprint pattern, consistent with the color and light conditions of real contactless samples. The usability of the produced virtual images for the design of new recognition algorithms and biometric evaluations has been proved by applying standard reconstruction methods and minutiae extraction techniques. However, a realistic modeling of the finger skin color must be improved.

\section{REFERENCES}

[1] R. Donida Labati, A. Genovese, V. Piuri, and F. Scotti, "Virtual environment for 3-D synthetic fingerprints," in 2012 IEEE International Conference on Virtual Environments, Human-Computer Interfaces and Measurement Systems, 2012.

[2] S. Ferrari, V. Piuri, and F. Scotti, "Virtual environment for granulometry analysis," in IEEE International Conference on Virtual Environments, Human-Computer Interfaces and Measurement Systems, 2008.

[3] A. Genovese, R. Donida Labati, V. Piuri, and F. Scotti, "Virtual environment for synthetic smoke clouds generation," in IEEE International Conference on Virtual Environments, Human-Computer Interfaces and Measurement Systems, 2011.

[4] G. Parziale and Y. Chen, "Advanced technologies for touchless fingerprint recognition," in Handbook of Remote Biometrics, ser. Advances in Pattern Recognition, M. Tistarelli, S. Z. Li, and R. Chellappa, Eds. Springer London, 2009, pp. 83-109.

[5] G. Parziale, E. Diaz-Santana, and R. Hauke, "The surround imager: A multi-camera touchless device to acquire 3D rolled-equivalent fingerprints." in ICB'06, 2006, pp. 244-250.

[6] Y. Wang, L. Hassebrook, and D. Lau, "Data acquisition and processing of 3-D fingerprints," IEEE Transactions on Information Forensics and Security, vol. 5, no. 4, pp. 750-760, December 2010.

[7] R. Donida Labati, A. Genovese, V. Piuri, and F. Scotti, "Fast 3D fingertip reconstruction using a single two-view structured light acquisition," in 2011 IEEE Workshop on Biometric Measurements and Systems for Security and Medical Applications (BioMS), 2011, pp. 1-8.

[8] R. Donida Labati, A. Genovese, V. Piuri, and F. Scotti, "Two-view contactless fingerprint acquisition systems: a case study for clay artworks," in Proc. of the 2012 IEEE Workshop on Biometric Measurements and Systems for Security and Medical Applications (BioMS), 2012.

[9] J. Araque, M. Baena, B. Chalela, D. Navarro, and P. Vizcaya, "Synthesis of fingerprint images," in 16th International Conference on Pattern Recognition, vol. 2, 2002, pp. 422-425.

[10] M. Kücken and A. C. Newell, "A model for fingerprint formation," $E P L$ (Europhysics Letters), vol. 68, no. 1, p. 141, 2004.

[11] M. Kücken, "Models for fingerprint pattern formation," Forensic Science International, vol. 171, no. 2-3, pp. 85-96, 2007.

[12] U.-K. Cho, J.-H. Hong, and S.-B. Cho, "Automatic fingerprints image generation using evolutionary algorithm," in Proceedings of the 20th international conference on Industrial, engineering, and other applications of applied intelligent systems, ser. IEA/AIE'07. Berlin, Heidelberg: Springer-Verlag, 2007, pp. 444-453.

[13] R. Cappelli, "Synthetic fingerprint generation," in Handbook of Fingerprint Recognition, 2nd ed., D. Maltoni, D. Maio, A. Jain, and S. Prabhakar, Eds. Springer London, 2009, pp. 271-301.

[14] J. Xue, S. Xing, Y. Guo, and Z. Liu, "Fingerprint generation method based on gabor filter," in 2010 International Conference on Computer Application and System Modeling (ICCASM), October 2010, pp. 115119.

[15] Q. Zhao, A. Jain, N. Paulter, and M. Taylor, "Fingerprint image synthesis based on statistical feature models," in 2012 IEEE Fifth International Conference on Biometrics: Theory, Applications and Systems (BTAS), 2012, pp. 23-30.

[16] D. Maltoni, D. Maio, A. K. Jain, and S. Prabhakar, Handbook of Fingerprint Recognition, 2nd ed. Springer Publishing Company, Incorporated, 2009.

[17] R. Donida Labati, A. Genovese, V. Piuri, and F. Scotti, "Contactless fingerprint recognition: a neural approach for perspective and rotation effects reduction," in IEEE Workshop on Computational Intelligence in Biometrics and Identity Management, 2013.

[18] L. Hong, Y. Wan, and A. Jain, "Fingerprint image enhancement: algorithm and performance evaluation," IEEE Transactions on Pattern Analysis and Machine Intelligence, vol. 20, no. 8, pp. 777-789, August 1998.

[19] Blender, http://www.blender.org/.

[20] C. I. Watson, M. D. Garris, E. Tabassi, C. L. Wilson, R. M. McCabe, S. Janet, and K. Ko, "User's guide to NIST biometric image software (NBIS)," National Institute of Standards and Technology, January 2007, http://fingerprint.nist.gov/NFIS. 